and to all outward appearances quite healthy; he complained of no pain and lay on the couch with his legs fully extended. His pulse and respirations were normul and his temperature was $99^{\circ} \mathrm{F}$. The abdominal muscles were kept somewhat rigid and made palpation difficult, but at a point just above, and to the left of, the umbilicus and along the outer border of the left rectus there appeared to be a rounded tumour, which, however, was felt for only a moment, disappearing almost immediately from under the fingers. Not satisfied with this examination, I asked one of my colleagues to administer chloroform in order that I might make a more thorough examination, with the result that a definite tumour, measuring about three inches by one and a half inches, somewhat reniform in shape and freely moveable, was felt lying just ahove, and chiefly to the left of, the umbilicus. On rectal examination nothing abnormal was detected and on withdrawing the examining finger the child passed a loose stool. There was no blood or mucus.

The boy was admitted into the hospital and passed a comfortable night and on the next morning had two stools, both quite normal and well formed. During that day, however, he vomited repeatedly. He was seen that afternoon by Mr. Ewen C. Stabb who thought that the abdominal tumour was probably due to a mass of enlarged mesenteric glands, the vomiting being accounted for by the fact that the boy had been given chloroform a few hours previously. The symptoms not callir $\mathrm{g}$ for immediate operation Mr. Stabb decided to explore the abdomen on the following day.

Under chloroform laparotomy was performed and the case proved to be one of intussusception. It was easily reduced with the exception of the last portion (about one and a half inches) which was œdematous and consisted of the appendix deeply imbedded in a fold in the wall of the cæcum firmly bound down by adhesions, and this, it would appear, had formed the apex or head of the intussusceptum. On "freeing" the appendix it was found to be in a chronic inflammatory condition and to have perforated about one inch from its base. Appendicectomy was purformed and the abdominal incision was closed. The boy made an uninterrupted recovery and was discharged from the hospital on Nov. 6th.

Judging from the condition found at the operation the child had doubtless been suffering from appendicitis during the early part of his illness, adhesions had formed completely burying the appendix, the resulting tumour protruding into the lumen of the gut, and it is reasonable to suppose that this had acted as a foreign body and had been the cause of the intussusception.

I am indebted to Mr. Stabb for permission to publish this case.

\section{A CASE OF CANCER IN THE MALE BREAST.}

By G. Ernest Peacheli, M.B., B.S. Lond., M.R.C.S. Eng., L R.C.P. LOND.

ASSISTANT MEDIOAL OFFICER, WEST SUSSEX ASTLUM.

CANCER of the breast being of rare occurrence in the male subject the following case may prove of interest. The patient, who was a man, aged 58 years, was admitted into the West Susiex Cuunty Asylum on Feb. 21st, 1905, suffering from melancholia with distressing auditory and visual hallucinations. He was tall, fat, and well nourished; he had a double inguinal hernia, and his cardiac sounds were rather feeble. Both breasts were more developed than normal and situated in the left one, below and internal to the nipple, there was a small irregular soft lump, nonadherent to the skin or underlying structures. There was a blood-stained discharge on squeezing the nipple but no glandular enlargement and the rest of the breast was healthy. The patient stated that the tumour had been gradually forming for two years but had given him no pain or trouble. The condition was diagnosed as possibly a "duct papilloma." The growth steadily increased in size and in January, 1906, it was noted to be " an irregular hard mass, adherent to the skin, which is thin, blue, and $c$ ngested over it and fixed to the pectoral fascia. The blood discharge continues from the nipple, which is slightly retracted. No glands to be felt in axilla." As the growth was evidently malignant and would have so $n$ ulcerated through the skin I removed the breast and the lower part of the pectoral muscle on Jan. 23rd. The patient marle a good recovery from this, but continued activtly suicidal, agitated and depressed, and died on Sept. 10th, from fatty heart and syncope. At the necropsy there was no sign of secondary deposits in any of the organs. The growth was invading the pectoral muscle and the microscope showed it to be a ty pical scirrhous carcinoma. It seemed strange that, considering the growth and ts activity, the axillary glands remained unaffected.

I am indebted to Mr. H. A. Kidd, the medical superintendent, for permission to publish this case.

Chichester.

\section{Wtedical Sorteties.}

\section{ROYAL MEDICAL AND CHIRURGICAL SOCIETY.}

Adjourned Disoussion on the Operative Treatment of NonMalignant Uloer of the Stomaoh and its Chief Complica. tions wi.h Indisations, Limitations, and Ultimate Results.

A Meeting of this society was beld on Dec. 11th, Dr. Thomas Buzzard, Vice-President, being in the chair.

Mr. H. GILBERT BARLING said that the complete and well. balanced address by Mr. Mayo Robson in opening the discussion left little more to be expressed and certainly very little in the way of criticism. He (Mr. Barling) was not at present convinced of the wisdom or necessity of gastro-jejunostomy as a general procedure in the presence of perforation. It might be required after recovery from the emergency operation but would in many cases when performed at the same time prejudice gravely the chance of recovery, and he illustrated this from cases upon which he had operated at a later stage. In his opinion the great factor which dominated all others in recovery after perforation was the briefness of the time elapsing between the calamity and surgical intervention. He was not prepared at present from his own observation to accept gastric ulcer as a common provocation of cancer, but he thought that this question deserved very careful investigation. Mr. Barling then referred to his own recent experiences of gastric surgery as shown in 29 cases operated on during the preceding year. Three of these patients suffered from hour-glass contraction, 17 from stenosis at or about the pylorus, sometimes complicated with active ulceration, three from ulcer with adhesions due to old perforation, and six from old-standing ulcer without complication. The operation of election in these cases was posterior gastro-jejunostomy and this was performed with but few exceptions, all the patients recovering. Whilst the results were generally thoroughly satisfactory he had to acknowledge one complete failure and two others in which relief was partial only. He referred to the difficulty of deciding when to operate for bæmatemesis and would greatly hesitate to intervene in young women with profuse bleeding in whom the prior history gave little evidence pointing to gastric ulcer. The difficulty of diagnosing gastric ulcer should not be lightly dismissed and this point was illustrated from recent experiences. Stenosis was more readily determined and when dilatation of the stomach with stasis was found justification for operation was established. The statistics quoted to show the small mortality from gastric ulcer were not very convincing, partily from the uncertainty of diagnosis and partly from the predominance of female cases in these statistics, whereas his own operations showed a considerable predominance of male patients. Statistics also failed to show the misery and inability to work which resulted from ulcer. Finally he urged the wisdom of a reasonable use of exploratory operation in long-standing doubtful cases.

Dr. E. I. SPRIGGS said that there was a general agreement that a number of complications of gastric ulcer could only be effiviently treated by operation. A claim had also been put forward to include among these hæmorrhage if severe or recurrent, and those cases which under ordinary medical treatment had relapsed. Such a claim to be established must be supported by statistics giving detailed accounts of the cases with special reference to the after-history. In looking through the one or two published series in which details were given he had found very few cases going back more than two or three years. Without prejudice to what the future might show, he thought that it was not justifiable to recommend operation because a case had relapsed until a fuller experience of the after-history of patients treated by gastro-enterostomy had been gained. He had not found any 